\title{
Indicadores de sustentabilidade - um levantamento dos principais sistemas de avaliação
}

\author{
Hans Michael Van Bellen *
}

\begin{abstract}
Resumo
Este artigo apresenta os resultados de um levantamento cujo objetivo é determinar as principais metodologias de avaliação para mensurar a sustentabilidade do desenvolvimento. Para isso, discute a crise ambiental e suas principais conseqüências, até o surgimento do conceito de desenvolvimento sustentável. Esse conceito, por sua vez, é observado a partir de diferentes abordagens que visam evidenciar a necessidade de um conhecimento mais aprofundado dos diferentes sistemas de indicadores de sustentabilidade e de sua utilização.

Sistemas de indicadores de desenvolvimento sustentável podem constituir uma importante ferramenta de gestão ambiental, pública ou privada, à medida que consigam orientar e mensurar o desenvolvimento. Entretanto, para alcançar esse objetivo, é necessário conhecer melhor as metodologias existentes. Nesse sentido, o primeiro passo para conhecer melhor as ferramentas de avaliação do desenvolvimento é determinar quais as mais importantes em termos de reconhecimento internacional.
\end{abstract}

Palavras-chave: desenvolvimento sustentável; indicadores de sustentabilidade; meio ambiente e sociedade.

\begin{abstract}
This article presents the results of a survey which aims to determine the most important methods of evaluation to measure the sustainability of development. In this sense, the text discusses the environment crisis and its main consequences up to the appearance of the concept of sustainable development. This concept is seen considering different approaches in order to emphasizes the need for enhancing the knowledge about different sustainability indicators systems and their use. Systems of sustainable development indicators may be important tools for public or private environmental management as they are able to guide and measure the development. However, it is important a more detailed comprehension on the existing methodologies concerning to sustainable development. The first step towards a better comprehension on the sustainability evaluation tools is determining which of these tools are the most important in terms of international acknowledgment.
\end{abstract}

Keywords: sustainable development; sustainability indicators; environment and society.

\section{Introdução}

O breve século XX, como afirma Hobsbawm (1996), foi testemunha de transformações significativas em todas as dimensões da existência humana. Ao lado do exponencial desenvolvimento tecnológico - que aumenta a expectativa de vida dos seres humanos ao mesmo tempo em que aumenta sua capacidade de autodestruição ocorreu um crescimento significativo da utilização de matéria e energia para atender às necessidades da sociedade. Essa demanda por bens e serviços é mundial, mas a pressão que exerce sobre o planeta não é uniforme. Observa-se uma grande disparidade quanto ao padrão de vida e de consumo das populações de diferentes países, juntamente com índices de desigualdade crescentes dentro desses países. A reflexão sobre o tema desenvolvimento, juntamente com o aumento da pressão exercida pela antroposfera sobre a ecosfera, levou ao crescimento da consciência acerca dos problemas ambientais gerados por padrões de vida incompatíveis com o processo de regeneração do meio ambiente. " Doutor pela Universidade Federal de Santa Catarina, Programa de Pós- Graduação em Engenharia da Produção e Sistemas. Professor - Pesquisador do Curso de Pós-
Graduação em Administração da Universidade Federal de Santa Catarina (CPGA/UFSC).

Artigo recebido em outubro de 2003 e aceito em dezembro de 2003. 
Essa reflexão, que começa a surgir a partir da década de 1970, vai levar ao surgimento do conceito de desenvolvimento sustentável. Tal conceito preconiza um tipo de desenvolvimento que garanta qualidade de vida para as gerações atuais e futuras, sem a destruição da sua base de sustentação, que é o meio ambiente. O surgimento do termo desenvolvimento sustentável, que se tornou rapidamente uma unanimidade em todos os segmentos da sociedade, ocasionou o aprofundamento da discussão sobre o real significado teórico e prático desse conceito. A questão que se estabelece a partir desse aprofundamento é: como o desenvolvimento sustentável pode ser definido e operacionalizado, para que seja utilizado como ferramenta de ajuste dos rumos que a sociedade vem tomando em relação a sua interação com o meio ambiente?

Este estudo procura preencher essa lacuna, sendo parte de um projeto maior que visa a uma compreensão mais aprofundada a respeito desse tema complexo. Por sua vez, o objetivo central desse projeto é fazer uma análise comparativa das ferramentas mais relevantes em termos de avaliação de sustentabilidade, e este artigo aborda a primeira etapa desse trabalho. Nessa primeira etapa, o objetivo principal é conhecer os principais sistemas de indicadores de sustentabilidade desenvolvidos e utilizados atualmente na comunidade internacional.

Para abordar esse tema, este artigo foi estruturado da seguinte forma: a primeira parte aborda sucintamente o referencial teórico que serviu de subsídio para este trabalho. São discutidos o reconhecimento da crise ambiental, o surgimento do conceito de desenvolvimento sustentável, as diferentes abordagens referentes a esse conceito, os indicadores de sustentabilidade e, por último, sua utilização como ferramenta para a gestão ambiental. Na segunda parte é apresentado o método de trabalho utilizado, e na terceira e última parte são apresentados e discutidos os resultados obtidos neste levantamento.

\section{Referencial teórico}

\section{Crise ambiental e o surgimento do conceito de desenvolvimento sustentável}

As crescentes dúvidas em relação ao futuro do meio ambiente são uma das consequiências das várias transformações que marcaram a segunda metade do século XX. Entre os anos 1960 e 1980, vários desastres ambientais como o da Baía de Minamata, no Japão, o acidente de Bhopal, na Índia, e o acidente na usina nuclear de Chernobyl, na extinta União Soviética, provocaram na Europa um impressionante avanço na conscientização sobre os problemas ambientais. O vazamento de petróleo do Exxon Valdez teve o mesmo impacto na América do Norte, provocando imensa irritação popular nos EUA.

Já a noção de desenvolvimento sustentável tem sua origem, mais remota, no debate internacional acerca do conceito de desenvolvimento. Na verdade, trata-se da história da reavaliação da noção de desenvolvimento predominantemente ligado à idéia de crescimento - até o surgimento do conceito de desenvolvimento sustentável. Segundo Brüseke (1995), alguns pontos importantes na discussão desse conceito foram: o relatório sobre os limites do crescimento, publicado em 1972; o surgimento do conceito de ecodesenvolvimento, em 1973; a declaração de Cocoyok, em 1974; o relatório da Fundação Dag-Hammarskjöld, em 1975, e finalmente a Conferência da Organização das Nações Unidas sobre Meio Ambiente e Desenvolvimento, em 1992, no Rio de Janeiro.

O primeiro impacto foi produzido pelo Clube de Roma - uma associação de cientistas, políticos e empresários preocupados com algumas questões globais -, que encomenda projetos relacionados a essas questões. Em 1972 surge um dos mais famosos estudos decorrentes dessa ação do Clube de Roma, o relatório mundialmente conhecido como "The limits to growth" (MEADOWS, 1972).

Esse relatório foi publicado em 1972, o mesmo ano em que foi realizada uma conferência em Estocolmo, na Suécia, sobre o meio ambiente humano. $\mathrm{O}$ documento ressaltava que a maioria dos problemas ligados ao meio ambiente ocorriam em escala global e se aceleravam de forma exponencial. O relatório rompeu com a idéia de ausência de limites para exploração dos recursos naturais, em clara contraposição à idéia dominante de crescimento contínuo da sociedade industrial. Durante a Conferência de Estocolmo, a preocupação principal, portanto, foi o crescimento populacional, bem como o processo de urbanização e a tecnologia envolvida na industrialização. 
Em 1973, surge pela primeira vez o termo ecodesenvolvimento, colocado como alternativa à concepção clássica de desenvolvimento. Alguns dos aspectos principais para formulação desse novo modelo foram articulados por Ignacy Sachs (1997). Esses aspectos abordavam prioritariamente a questão da educação, da participação e da preservação dos recursos naturais juntamente com a satisfação das necessidades básicas. $\mathrm{O}$ conceito de ecodesenvolvimento se referia inicialmente a algumas regiões de países subdesenvolvidos e foi um grande avanço na percepção do problema ambiental global, na medida em que se começou a perceber a interdependência entre desenvolvimento (ou seu modelo dominante) e meio ambiente.

Em 1974, é formulada a Declaração de Cocoyok, resultado de uma reunião da Conferência das Nações Unidas sobre Comércio e Desenvolvimento e do Programa de Meio Ambiente das Nações Unidas. O documento inovou na discussão sobre desenvolvimento e meio ambiente, uma vez que lançava algumas hipóteses sobre a relação entre esses dois elementos. O documento afirma que:

- a explosão populacional é decorrente da absoluta falta de recursos em alguns países (quanto maior a pobreza, maior é o crescimento demográfico);

- a destruição ambiental também decorre da pobreza e

- os países desenvolvidos têm uma parcela de culpa nos problemas globais, uma vez que apresentam elevado nível de consumo.

Em 1975, a Fundação Dag-Hammarskjöld aprofunda as conclusões da declaração de Cocoyok, publicando um relatório que contou com a colaboração de 48 países, juntamente com o Programa de Meio Ambiente das Nações Unidas e outras 13 organizações da ONU. O relatório se concentra na questão do poder e sua relação com a degradação ambiental, destacando o papel de um novo desenvolvimento baseado na mobilização das forças capazes de mudar as estruturas dos sistemas vigentes.

Em 1992, 20 anos depois da reunião pioneira de Estocolmo, uma nova conferência da ONU sobre meio ambiente e desenvolvimento é realizada, no Rio de Janeiro, aumentando o grau de consciência sobre o modelo de desenvolvimento adotado mundialmente, e, também, sobre as limitações que este apresenta. Finalmente, a interligação entre desenvolvimento socioeconômico e as transformações do meio ambiente entram no discurso oficial da maioria dos países do mundo. A entendimento quanto à relação entre problemas ambientais e o processo de desenvolvimento se legitima através do surgimento do conceito de desenvolvimento sustentável (GUIMARÃES, 1997).

\section{As diferentes abordagens do desenvolvimento sustentável}

Como já foi observado, o conceito de desenvolvimento sustentável resulta de um relativamente longo processo histórico de reavaliação crítica da relação entre a sociedade civil e seu meio natural. Por se tratar de um processo contínuo e complexo, hoje existem várias abordagens que procuram explicar o conceito de sustentabilidade. Essa variedade pode ser constatada pelas inúmeras definições relativas a esse conceito.

O termo desenvolvimento sustentável foi primeiramente discutido pela World Conservation Union, também chamada de International Union for the Conservation of Nature and Natural Resources (IUCN), no documento intitulado "World's conservation strategy" (IUCN, 1980). De acordo com esse documento, para que o desenvolvimento seja sustentável, devem ser considerados aspectos referentes às dimensões social e ecológica, bem como fatores econômicos, recursos vivos e não-vivos e as vantagens de curto e longo prazo de ações alternativas. O foco do conceito está centrado na integridade ambiental, e apenas a partir da definição do Relatório Brundtland, a ênfase se desloca para o elemento humano, gerando um equilíbrio entre as dimensões econômica, ambiental e social.

O Relatório Brundtland - elaborado a partir da World Commission on Environment and Development (WCED) - traz uma das definições mais conhecidas, segundo a qual o desenvolvimento sustentável é aquele que atende às necessidades das gerações presentes, sem comprometer a possibilidade das gerações futuras atenderem suas próprias necessidades (WORLD COMMISSION ON ENVIRONMENT AND DEVELOPMENT, 1987). 
Para Goldsmith (1972), uma sociedade pode ser considerada sustentável quando todos os seus propósitos e intenções podem ser atendidos indefinidamente, garantindo plena satisfação aos seus membros. Pronk (1992) destaca o papel do crescimento econômico na sustentabilidade. Para ele, o desenvolvimento é sustentável quando o crescimento econômico traz justiça e oportunidades para todos os seres humanos do planeta, sem privilégio de algumas espécies, sem destruir os recursos naturais finitos e sem ultrapassar a capacidade de carga do sistema.

Para algumas organizações não-governamentais, e para o próprio Programa das Nações Unidas em Meio Ambiente e Desenvolvimento, o desenvolvimento sustentável consiste na modificação da biosfera e na aplicação de seus recursos, para atender às necessidades humanas e aumentar a qualidade de vida (IUCN/UNEP/WWF, 1980). Para que esse desenvolvimento seja sustentável, devem ser considerados os fatores social, ecológico e econômico, dentro de perspectivas de curto, médio e longo prazo.

Segundo Costanza (1991), o conceito de desenvolvimento sustentável deve ser inserido na relação dinâmica entre o sistema econômico humano e um sistema maior, com taxa de mudança mais lenta, o ecológico. Para ser sustentável, essa relação deve assegurar que a vida humana possa crescer e desenvolver sua cultura, indefinidamente, observando-se que os efeitos das atividades humanas permaneçam dentro de fronteiras adequadas, de modo a não destruir a diversidade, a complexidade e as funções do sistema ecológico de suporte à vida. Munasinghe e McNeely (1995) resumem a sustentabilidade na obtenção de um conjunto de indicadores que sejam referentes ao bem-estar, que possam ser mantidos ou que cresçam no tempo.

O termo desenvolvimento sustentável pode ser visto como uma palavra-chave, considerando que existem numerosas definições para esse conceito. As definições comumente mais conhecidas, citadas e aceitas são a do Relatório Brundtland (WORLD COMMISSION ON ENVIRONMENT AND DEVELOPMENT, 1987) e o documento conhecido como "Agenda 21". A mais conhecida definição do Relatório Brundtland apresenta a questão das gerações futuras e suas possibilidades. Essa definição contém dois conceitos-chave: o conceito de necessidade - referindo-se particularmente às necessidades dos países mais subdesenvolvidos - e a idéia de limitação, imposta pelo estado da tecnologia e de organização social, para atender às necessidades do presente e do futuro.

A questão da ênfase do componente social no desenvolvimento sustentável se reflete no debate sobre a inclusão ou não de medidas sociais, na definição. Esse debate resulta da variedade de concepções de sustentabilidade que contêm componentes não usualmente mensurados, como o cultural e o histórico. Os indicadores sociais são considerados especialmente controversos, pois refletem contextos políticos e julgamentos de valor. A integração de medidas é ainda mais complicada em função das diferentes - e muitas vezes incompatíveis dimensões da sustentabilidade. A definição do Relatório Brundtland não estabelece um estado estático, mas um processo dinâmico, que pode continuar a existir sem a lógica autodestrutiva predominante. As diferentes forças que atuam no sistema devem estar em balanço para que o sistema como um todo se mantenha no tempo.

Além da definição fornecida pelo Relatório Brundtland, existem muitas outras definições do que seja desenvolvimento sustentável. Apesar dessa grande quantidade de definições, ou talvez devido exatamente a esse fato, não se sabe com exatidão o que o termo significa. Mas não é o objetivo deste trabalho identificar a maioria dessas definições de sustentabilidade (que para alguns autores chega a 160), mas sim verificar como varia o entendimento do que seja sustentabilidade. Os objetivos do desenvolvimento sustentável desafiam as instituições contemporâneas. Estas têm reagido às mudanças globais, relutando em reconhecer que esse processo esteja realmente ocorrendo. As diferenças em relação ao conceito de desenvolvimento sustentável são tão grandes que não existe consenso sobre o que deve ser sustentado e tampouco sobre o que o termo "sustentar" significa. Conseqüentemente, não existe consenso sobre como medir a sustentabilidade. Infelizmente, para a maioria dos autores anteriormente citados, sem uma definição operacional minimamente aceita, torna-se impossível traçar estratégias e acompanhar o sentido e a direção do progresso.

Todas as definições e ferramentas relacionadas à sustentabilidade devem considerar o fato de que não se conhece totalmente como o sistema opera. Pode-se apenas descobrir os impactos ambientais decorrentes de atividades e a interação com o bem-estar humano, com a economia e com o meio ambiente. Em geral, sabe-se que o sistema interage entre as diferentes dimensões mas não se conhece especificamente o impacto dessa interação. 
Todos esses aspectos já apresentados mostram a diversidade e a complexidade do termo desenvolvimento sustentável. Apesar da dificuldade que essas características conferem ao objeto de estudo, essa diversidade de conceituação deve servir não como obstáculo na procura de um melhor entendimento, mas sim como fator de motivação e de criação de novas visões acerca de ferramentas que procurem descrever a sustentabilidade.

\section{Indicadores de desenvolvimento sustentável}

O termo indicador é originário do latim indicare, que significa descobrir, apontar, anunciar, estimar (HAMMOND, 1995). Os indicadores podem comunicar ou informar sobre o progresso em direção a uma determinada meta, como por exemplo o desenvolvimento sustentável, mas também podem ser entendidos como um recurso que deixa mais perceptível uma tendência ou fenômeno, que não seja imediatamente detectável (HAMMOND, 1995).

A definição de McQueen e Noak (1988) trata um indicador como uma medida que resume informações relevantes de um fenômeno particular ou um substituto dessa medida, semelhante ao conceito de Holling (HOLLING, 1978) de que um indicador é uma medida do comportamento do sistema, em termos de atributos expressivos e perceptíveis.

Para a Organização para a Cooperação e Desenvolvimento Econômico (OCDE), um indicador deve ser entendido como um parâmetro - ou valor derivado de parâmetros - que aponta e fornece informações sobre o estado de um fenômeno com uma extensão significativa (OECD, 1993).

Algumas definições colocam um indicador como uma variável relacionada hipoteticamente a uma outra variável estudada, que não pode ser diretamente observada (CHEVALIER, 1992). Essa também é a opinião de Gallopin (1996), que afirma que os indicadores, num nível mais concreto, devem ser entendidos como variáveis.

Uma variável é uma representação operacional de um atributo (qualidade, característica, propriedade) de um sistema. A variável não é o próprio atributo ou atributo real, mas uma representação, imagem ou abstração deste. O quão próximo essa variável se aproxima do atributo próprio ou reflete o atributo ou a realidade, e qual o seu significado ou qual a sua significância e relevância para a tomada de decisão, é conseqüência da habilidade do investigador e das limitações e propósitos da investigação.

Nesse contexto, qualquer variável, e conseqüentemente qualquer indicador (descritivo ou normativo), tem uma significância própria. A mais importante característica do indicador, quando comparado com os outros tipos ou formas de informação, é a sua relevância para a política e para o processo de tomada de decisão. Assim, para ser representativo, o indicador tem que ser considerado importante tanto por quem toma decisões quanto pelo público (GALLOPIN, 1996).

Segundo Gallopin (1996), os indicadores mais desejados são aqueles que resumam ou simplifiquem as informações relevantes, façam com que certos fenômenos que ocorrem na realidade se tornem mais aparentes; aspecto esse que é particularmente importante na gestão ambiental. Nessa área, especificamente, é necessário que sejam quantificadas, mensuradas e comunicadas as ações relevantes.

Como foi anteriormente observado, a emergência da temática ambiental está fortemente relacionada à falta de percepção da ligação existente entre ação humana e suas principais conseqüências; no caso específico deste trabalho, à degradação ambiental.

Para Tunstall (1992, 1994), os indicadores devem ser observados a partir de suas funções, que são:

- avaliar condições e tendências;

- efetuar a comparação entre lugares e situações;

- avaliar condições e tendências em relação às metas e objetivos;

- prover informações de advertência;

- antecipar futuras condições e tendências. 
O objetivo principal dos indicadores é o de agregar e quantificar informações de uma maneira que sua significância fique mais aparente. Os indicadores simplificam as informações sobre fenômenos complexos tentando, com isso, melhorar o processo de comunicação.

\section{Indicadores de sustentabilidade: uma ferramenta para a gestão ambiental}

A Conferência Internacional da Organização das Nações Unidas sobre Meio Ambiente e Desenvolvimento, realizada no Rio de Janeiro, adotou a Agenda 21 para transformar o desenvolvimento sustentável numa meta global aceitável. Para colocar os princípios da sustentabilidade em prática e adotar os princípios da Agenda 21, essa conferência criou a Comissão de Desenvolvimento Sustentável (CSD - Comission on Sustainable Development), cuja principal responsabilidade é a de monitorar o progresso alcançado.

A necessidade de se consolidar indicadores de desenvolvimento sustentável está expressa na própria Agenda 21, nos capítulos 8 e 40. A partir da conferência no Rio de Janeiro, a CSD adotou um programa de cinco anos para o desenvolvimento de instrumentos que sejam adequados aos que tomam decisões a nível nacional.

Um dos principais aspectos levantados nos primeiros encontros da CSD foi o da necessidade de se criar padrões que sirvam de referência para medir o progresso da sociedade em direção ao que se convencionou chamar de futuro sustentável (MOLDAN e BILHARZ, 1997). É necessário trabalhar com uma unidade que possibilite medir a proximidade em relação a esse objetivo. E essa unidade deve ser suficientemente ampla para englobar uma gama de fatores relacionados com a sustentabilidade, tais como fatores ecológicos, econômicos, sociais, culturais, institucionais, entre outros.

Em termos do conceito de desenvolvimento sustentável, deve-se observar que a abordagem pode ser feita a partir de diversos níveis ou esferas específicas. Em termos geográficos é possível abordar o conceito na esfera mundial, nacional, regional e local. Em relação ao aspecto temporal, pode se abordar a curto, a médio ou a longo prazo; e quanto aos atores envolvidos, a ênfase pode ser atribuída ao indivíduo, ao grupo ou à sociedade. Entretanto, segundo a CSD, existe a necessidade de se criar uma base comum para que se tenha um denominador para avaliação do grau de sustentabilidade, uma vez que a maioria dos indicadores existentes não são adequados para isso.

As ferramentas de avaliação também são úteis para quem toma decisões, na medida em que podem ser utilizadas para o desenvolvimento de políticas, na função de planejamento, levando-se em conta, ainda, que esses tipos de ferramentas cumprem outras funções:

- função analítica - as medidas ajudam a interpretar os dados dentro de um sistema coerente, agrupando-os em matrizes ou índices;

- função de comunicação - as ferramentas tornam os tomadores de decisão familiarizados com os conceitos e métodos envolvidos na sustentabilidade. Os indicadores ajudam no estabelecimento de metas e também na avaliação do sucesso em alcançá-las;

- função de aviso e mobilização - as medidas auxiliam os administradores a colocarem os mecanismos de uma forma pública, publicações anuais ou simples relatórios com indicadores-chave;

- função de coordenação - um sistema de medidas e de relatórios deve integrar dados de diferentes áreas e dados coletados por agências distintas. Deve ser factível, tanto em termos de orçamento como em termos de recursos humanos. Deve ser aberto à população, para participação e controle. Essas funções são melhor preenchidas no processo de escolha de indicadores e na fase de implementação, quando os tomadores de decisão utilizam as ferramentas de mensuração e os indicadores.

Observa-se que, apesar da existência de diversos sistemas relacionados à avaliação da sustentabilidade, existem diversos elementos que ainda não estão devidamente estudados e desenvolvidos. Pode-se recordar rapidamente alguns desses aspectos, tais como: a multidimensionalidade do conceito de desenvolvimento sustentável, a complexidade que decorre da agregação de variáveis não relacionadas diretamente, a questão da transparência em sistemas de avaliação, a existência dos julgamentos de valor e sua ponderação nos diversos sistemas, o tipo de processo decisório envolvido, bem como o tipo de variável envolvida (qualitativa, quantitativa ou as duas), entre outros. 
Teoricamente, é necessário organizar os diferentes sistemas, para que os usuários dessas ferramentas essenciais nos ajustes da direção do desenvolvimento - possam selecionar e trabalhar com os modelos mais adequados aos seus objetivos finais. E a finalidade deste trabalho, em sua primeira etapa, é justamente essa, fornecer um quadro sistematizado sobre as ferramentas de avaliação mais reconhecidas internacionalmente, que procuram mensurar o desenvolvimento em função da sustentabilidade.

\section{Método}

O referencial teórico deste artigo tratou de diversos aspectos relacionados às diferentes perspectivas do conceito de desenvolvimento sustentável. Nesse referencial, procurou-se explorar a relação do homem com o meio ambiente, a partir da tomada de consciência a respeito da crise ambiental que trouxe, como um dos seus resultados, o surgimento do conceito de desenvolvimento sustentável.

Os principais problemas relativos a esse conceito, com suas potencialidades e limitações, foram explorados para abordar as questões referentes às metodologias de avaliação da sustentabilidade. Como já foi exposto, existe um razoável grau de consenso no que se refere à necessidade de desenvolvimento de metodologias que permitam uma avaliação da sustentabilidade. Entretanto, as controvérsias a respeito do próprio conceito de desenvolvimento sustentável que inspira a construção dessas metodologias e de seus indicadores têm provocado inúmeras abordagens. Isso decorre de diferentes esquemas interpretativos relacionados ao conceito ordenador na formulação das ferramentas de avaliação, como também da complexidade do tema.

\section{Objetivos e delineamento da pesquisa}

Com o intuito de determinar quais as mais importantes ferramentas de avaliação de sustentabilidade existentes, foram estabelecidos os seguintes objetivos específicos:

- contextualizar o conceito de desenvolvimento sustentável;

- analisar os fundamentos teóricos e empíricos das ferramentas de avaliação de sustentabilidade;

- levantar as principais ferramentas de avaliação de sustentabilidade.

Para alcançar os objetivos propostos, optou-se por um delineamento do tipo descritivo exploratório, que segundo Cervo e Bervian (1996) procura observar, registrar, analisar e correlacionar fenômenos, sem sua manipulação. Por se tratar de estudo exploratório, não são estabelecidas hipóteses, apenas foram definidas metas que orientam a pesquisa.

Nessa etapa do projeto de pesquisa foram utilizados apenas dados primários. Estes foram obtidos a partir de um questionário enviado a uma amostra de pesquisadores e profissionais de diferentes organizações, oriundos das mais diversas áreas. $\mathrm{O}$ objetivo principal desse instrumento de coleta de dados foi selecionar as ferramentas mais conhecidas e relevantes de avaliação de sustentabilidade. Essa amostra é intencional, tendo em vista que os entrevistados escolhidos devem possuir conhecimentos ou alguma relação com iniciativas ligadas à sustentabilidade. Esse cuidado foi necessário, uma vez que as iniciativas ligadas a indicadores de sustentabilidade são pouco conhecidas. $O$ questionário utilizado era do tipo aberto, pois permitia ao entrevistado interagir com o en trevistador e sugerir iniciativas de avaliação que não estavam descritas no corpo do instrumento de coleta de dados.

\section{Técnica de coleta e análise dos dados}

A abordagem quantitativa predomina na primeira parte do trabalho. Um questionário foi enviado a cada um dos membros de uma amostra representativa de profissionais ligados a organizações dos setores público, privado e educacional, e da sociedade civil. Os dados obtidos foram submetidos a um tratamento prioritariamente quantitativo, com análise estatística das respostas, em que se procurou determinar as ferramentas mais conhecidas e importantes em termos de avaliação de sustentabilidade. 


\section{Justificativa e limitações da pesquisa}

A especificação do problema da pesquisa mostra que, atualmente, se faz necessário compreender melhor o conceito de desenvolvimento sustentável, suas características e limitações, para que se possa utilizar melhor esse sistema, em termos de orientação geral da sociedade. E para que se possa utilizar melhor esse conceito, deve-se conhecer as ferramentas existentes em temos de avaliação de sustentabilidade. Apesar do conceito de desenvolvimento sustentável ser relativamente novo, observa-se uma diversidade de abordagens na sua avaliação. Tal diversidade de abordagens está relacionada aos diferentes esquemas de interpretação relacionados ao conceito que gera uma variedade de ferramentas que lidam com sua avaliação. Nesse sentido, o processo de classificação e comparação de metodologias aparece como elemento necessário para orientar os diferentes atores sociais interessados na gestão ambiental. A classificação e a comparação de ferramentas de avaliação facilita a compreensão e o campo de aplicação dos diferentes sistemas.

Sistemas de indicadores de sustentabilidade são relevantes para o processo de gestão, na medida em que estão aptos a retratar a realidade de uma maneira científica, destinada a orientar na formulação de políticas. A classificação auxilia na identificação das principais vantagens e também das limitações dos diferentes processos de avaliação existentes; do mesmo modo que fornece uma revisão sistematizada dos métodos avaliados e comparados. A análise comparativa permite que diferentes grupos com diferentes objetivos e atuando em esferas diferenciadas tenham melhores condições de escolher e utilizar o método mais adequado para alcançar suas metas.

Apesar da grande importância que a seleção e análise comparativa de diferentes ferramentas pode trazer em termos de compreensão da realidade, deve-se observar que o presente trabalho apresenta algumas limitações. Uma dessas limitações está relacionada à seleção das metodologias a serem comparadas. O método não induzido, utilizado nessa seleção, deve levar a ferramentas que abordem diferentes dimensões relacionadas à sustentabilidade. Poderia ser realizada uma análise comparativa de métodos que trabalham dentro de dimensões específicas. Entretanto, por se tratar de um estudo exploratório e descritivo, e levando em conta o pequeno número de experiências de avaliação, optou-se por uma comparação generalizada das ferramentas de avaliação mais relevantes na atualidade.

\section{Descrição e análise dos resultados}

A seguir, serão descritos os resultados encontrados neste trabalho. O primeiro passo para a realização da análise comparativa de ferramentas de avaliação do desenvolvimento sustentável foi a seleção das metodologias consideradas mais importantes e promissoras em relação ao tema.

Inicialmente, o objetivo foi procurar determinar as principais ferramentas ou metodologias em desenvolvimento ou utilização referentes à avaliação de sustentabilidade. Essa primeira etapa consistiu de pesquisa bibliográfica, na qual se buscou a literatura relacionada ao tema "indicadores de desenvolvimento sustentável". Esse primeiro levantamento foi feito independentemente da área de atuação das diferentes ferramentas de avaliação, uma vez que o objetivo principal era conhecer as metodologias que têm sido mais estudadas e referidas. A partir desse levantamento, foi possível realizar a segunda etapa do projeto, a de identificação das principais ferramentas de avaliação.

\section{Seleção inicial}

O resultado do levantamento inicial conduziu a diversas iniciativas relacionadas à temática da sustentabilidade; e dentre todas as ferramentas pesquisadas, 18 diferentes métodos foram selecionados. Para essa seleção, foram utilizados dois critérios principais: o número de ocorrências e citações da ferramenta - entre os diversos artigos e relatórios pesquisados - e a existência de referencial teórico e empírico suficiente e adequado sobre o método. As ferramentas selecionadas nessa primeira etapa do trabalho, a partir dos critérios expostos, estão listadas no quadro 1. 
Quadro 1

Principais projetos em indicadores de desenvolvimento sustentável

\begin{tabular}{ll}
\hline PSR (Pressure/State/Response) & $\begin{array}{l}\text { OECD - Organization for Economic Cooperation and } \\
\text { Development }\end{array}$ \\
\hline DSR (Driving-Force/State/Response) & $\begin{array}{l}\text { UN/CSD - United Nations Comission on Sustainable } \\
\text { Development }\end{array}$ \\
\hline GPI (Genuine Progress Indicator & Cobb \\
\hline HDI (Human Development Index) & UNDP - United Nations Development Programme \\
\hline MIPS (Material Input per Service) & Wuppertal Institut - Alemanha \\
\hline DS (Dashboard of Sustainability) & $\begin{array}{l}\text { International Institut for Sustainable Development - } \\
\text { Canadá }\end{array}$ \\
\hline EFM (Ecological Footprint Model) & Wackernagel and Rees \\
\hline BS (Barometer of Sustainability) & IUCN - Prescott-Allen \\
\hline SBO (System Basic Orientors) & Bossel - Kassel University \\
\hline Wealth of Nations & (World Bank) \\
\hline SEEA (System of Integrating Environment and & United Nations Statistical Division \\
Economic) & \\
\hline NRTEE (National Round Table on the Environment and & Human/Ecosystem Approach - Canadá \\
Economy) & \\
\hline PPI (Policy Performance Indicator) & Holanda \\
\hline IWGSD (Interagency Working Group on Sustainable & US President Council on Sustainable Development \\
Development Indicators) & Indicator Set \\
\hline EE - Eco Efficiency & WBCSD (World Business Council on Sustainable \\
\hline SPI (Sustainable Process Index) & Development) \\
\hline EIP (European Indices Project & Institute of Chemical Engineering - Graz University \\
\hline ESI (Environmental Sustainability Index) & Eurostat \\
\hline & World Economic Forum \\
\hline
\end{tabular}

\section{Levantamento}

$\mathrm{Na}$ segunda etapa do projeto o objetivo era verificar, dentre as ferramentas de avaliação previamente escolhidas, quais as metodologias mais relevantes em relação ao objetivo de mensurar a sustentabilidade. Como anteriormente descrito, para isso foi utilizado um questionário do tipo aberto, enviado a diversos especialistas, de variados segmentos da sociedade, que têm trabalhado com temas relacionados à sustentabilidade. O processo de seleção da amostra dos especialistas foi realizado a partir da construção de uma lista com os principais participantes e palestrantes dos eventos internacionais mais importantes na área de desenvolvimento sustentável, e também de grupos interdisciplinares que trabalham nessa área. Um desses eventos foi o programa Science and Policy Dialogue", que organizou a conferência "Measure and communicate sustainable development", a qual foi dividida em duas partes: uma e-conference (conferência eletrônica) realizada entre 20 de fevereiro e 20 de março de 2001, baseada em documentos para discussão, e posteriormente uma conferência em Estocolmo, Suécia, em abril de 2001, organizada pela Swedish Environment Protection Agency e pela Foundation for Strategic Environmental Research (Mistra). A realização dessa conferência foi parte dos preparativos para a Conferência Rio+10, na busca das alternativas para melhor medir o progresso. Seus resultados constam do documento preparatório da reunião de cúpula de Gotemburgo, Suécia, realizada em junho de 2001.

Outro evento importante, utilizado para a seleção dos especialistas, foi o "Fourth international workshop on indicators of sustainable development", realizado na cidade de Praga, República Tcheca, em 1998. Também foi realizado um levantamento de especialistas ligados à sustentabilidade no grupo de pesquisa denominado Balaton Group", constituído por uma rede internacional de mais de 200 membros em aproximadamente 30 países. Trata-se de um grupo de estudos essencialmente interdisciplinar, que lida com temas variados, dentre os quais a relação entre sociedade e meio ambiente e as diferentes maneiras de se visualizar a sustentabilidade. 
Foram selecionados 80 especialistas que constituíram uma amostra de profissionais que atuam ou lidam com o tema da avaliação do desenvolvimento sustentável. Essa amostra foi dividida em quatro categorias específicas (categorias institucionais):

- organizações governamentais - 27 especialistas;

- organizações não-governamentais - 27 especialistas;

- instituições educacionais ou de pesquisa - 22 especialistas;

- instituições privadas - 4 especialistas.

Para cada um dos especialistas foi enviado um questionário, onde se solicitava a escolha - dentre as atuais metodologias conhecidas para avaliação do desenvolvimento - das cinco ferramentas que consideravam mais importantes e relevantes em termos de avaliação de sustentabilidade. Como orientação inicial, junto ao questionário, foi enviada uma lista com as 18 metodologias anteriormente selecionadas. Entretanto, o questionário utilizado deixava aberta a possibilidade do entrevistado adicionar quaisquer metodologias que pudessem ser consideradas relevantes.

\section{Análise dos resultados}

Um primeiro aspecto a ser observado é o grau de retorno dos questionários. Os questionários foram enviados apenas uma única vez para a amostra, e o grau de retorno foi elevado, sendo que dos 80 especialistas consultados, 45 enviaram uma resposta, ou um percentual de 56,25\%. Dentre os 45 respondentes, uma pequena parcela afirmou que não estava apta a selecionar (ou sugerir) metodologias, por não possuir conhecimento suficientemente adequado a respeito de todas elas. Uma parcela significativa dos especialistas também não sugeriu, especificamente, ferramentas mais relevantes, embora tenha enviado artigos e comentários relativos tanto ao conceito de desenvolvimento sustentável como alguns projetos relativos a indicadores. Uma outra parcela significativa respondeu sugerindo ferramentas que considerava mais importantes, sendo que nesse grupo, alguns respondentes enviaram comentários e artigos, enquanto outros apenas enumeraram as ferramentas mais relevantes. No gráfico 1 é possível observar a distribuição dos entrevistados que retornaram o questionário em relação ao tipo de resposta.

\section{Gráfico 1}

\section{Distribuição dos respondentes}
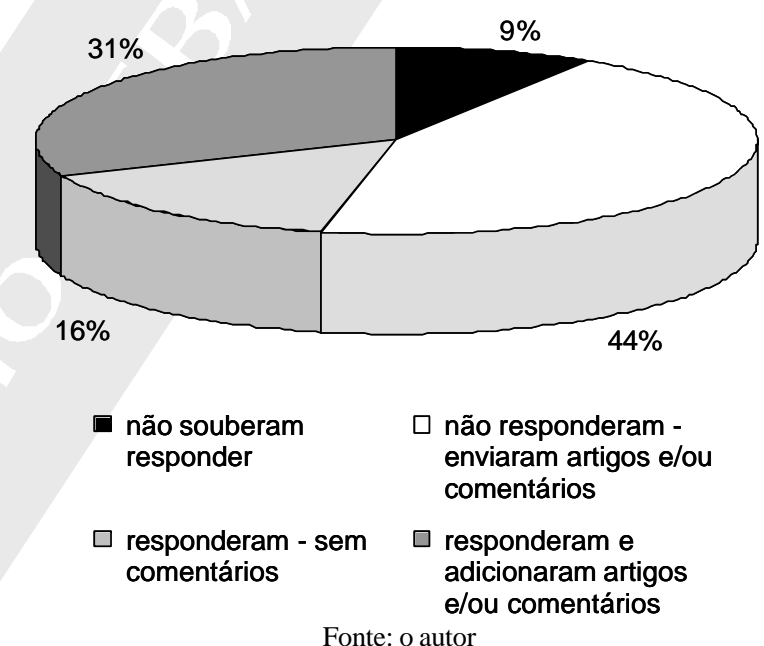

Nas tabelas 1 e 2 estão condensadas as informações sobre os respondentes em função da categoria institucional, grau de retorno e categoria de resposta. A observação dos dados contidos nessas tabelas permite algumas considerações. 
Na tabela 1, observa-se que o grau de retorno dos questionários dentro das diferentes esferas institucionais foi relativamente proporcional ao tamanho de cada uma das categorias. A única exceção se refere à categoria das organizações privadas ou comerciais, onde o grau de retorno foi nulo. Dentro da categoria instituições governamentais, o grau de retorno encontrado foi um pouco superior ao das outras duas categorias.

Tabela 1

Distribuição dos respondentes em função da categoria institucional e grau de retorno dos questionários

\begin{tabular}{|c|c|c|c|c|c|}
\hline \multirow[b]{2}{*}{ Categorias institucionais } & \multicolumn{2}{|c|}{ Enviados } & \multicolumn{2}{|c|}{ Respondidos } & \multirow{2}{*}{ Resposta na categoria $(\%)$} \\
\hline & Absoluto & $\%$ & Absoluto & $\%$ & \\
\hline Instituições educacionais ou de pesquisa & 22 & 27,50 & 11 & 24,44 & 50,00 \\
\hline Organizações não-governamentais & 27 & 33,75 & 15 & 33,33 & 55,56 \\
\hline Organizações governamentais & 27 & 33,75 & 19 & 42,22 & 70,37 \\
\hline Instituições privadas ou comerciais & 4 & 5,00 & 0 & 0,00 & 0,00 \\
\hline
\end{tabular}

Quando se consideram as categorias institucionais e as categorias de respostas (tabela 2), observa-se que proporcionalmente o menor índice de respostas específicas ficou na categoria das organizações governamentais, excetuando-se o caso das organizações privadas que, como já foi dito, não retornaram nenhum questionário. Entretanto, dentro da categoria das organizações governamentais, o índice de respostas não específicas mas com adição de artigos e comentários acerca dos sistemas existentes foi alto. Isso também ocorre com as categorias educacionais e não-governamentais.

Tabela 2

Distribuição dos respondentes em função da categoria de respostas e categoria institucional

\begin{tabular}{|c|c|c|c|c|c|c|c|}
\hline \multirow{2}{*}{ Categorias de respostas } & \multirow{2}{*}{ EDU } & \multirow{2}{*}{ ONGS } & \multirow{2}{*}{ GOV } & \multirow{2}{*}{$\mathrm{COM}$} & \multicolumn{3}{|c|}{ Percentual } \\
\hline & & & & & EDU & ONGS & GOV \\
\hline $\begin{array}{l}\text { Responderam especificamente e } \\
\text { adicionaram artigos e/ou comentários }\end{array}$ & $5(45,4 \%)$ & $5(33,3 \%)$ & $4(21,1 \%)$ & 0 & $35,71 \%$ & $35,71 \%$ & $28,57 \%$ \\
\hline $\begin{array}{l}\text { Responderam especificamente sem } \\
\text { adicionar comentários. }\end{array}$ & $1(9,1 \%)$ & $3(20,0 \%)$ & $3(15,8 \%)$ & 0 & $14,29 \%$ & $42,86 \%$ & $42,86 \%$ \\
\hline Não souberam responder & $2(18,2 \%)$ & $0(0,0 \%)$ & $2(10,5 \%)$ & 0 & $50,00 \%$ & $0,00 \%$ & $50,00 \%$ \\
\hline $\begin{array}{l}\text { Enviaram artigos e/ou comentários mas } \\
\text { não responderam especificamente }\end{array}$ & $3(27,3 \%)$ & $7(46,7 \%)$ & $10(52,6 \%)$ & 0 & $15,00 \%$ & $35,00 \%$ & $50,00 \%$ \\
\hline TOTAL & 11 & 15 & 19 & 0 & & & \\
\hline
\end{tabular}

GOV - organizações governamentais;

ONGS - organizações não governamentais;

EDU - instituições educacionais ou de pesquisa;

$\mathrm{COM}$ - instituições privadas.

O índice máximo dos que não souberam responder e não acrescentaram comentários teóricos sobre as ferramentas foi de $18,2 \%$ na categoria de instituições educacionais, sendo que o nível médio, considerando todos os tipos de instituições, foi de $9 \%$. O principal argumento para esse grupo (não souberam responder) foi o conhecimento de apenas algumas metodologias; por isso a incapacidade de selecionar as que consideram mais adequadas.

\section{Classificação das ferramentas de avaliação}

Para efeito de classificação das principais ferramentas de avaliação - classificação essa que será utilizada para o posterior estudo comparativo -, consideraram-se apenas os questionários que continham respostas específicas, com ou sem artigos e comentários técnicos adicionais. O material enviado pelos entrevistados que não responderam com indicações específicas foi utilizado para o aprofundamento da fundamentação teórica e para auxílio na construção das dimensões de análise. Os resultados obtidos com o questionário são apresentados na tabela 3. 
Os dados mostram que existe uma grande fragmentação entre os diversos especialistas, quanto às metodologias de avaliação de sustentabilidade que consideram mais importantes. Entretanto, as três metodologias mais lembradas cobrem juntas $35,4 \%$ das indicações. As metodologias que obtiveram mais indicações no levantamento foram:

EFM - Ecological Footprint Method;

DS - Dashboard of Sustainability;

BS - Barometer of Sustainability.

Tabela 3

Número de indicações obtidas por cada uma das ferramentas de avaliação de sustentabilidade

\begin{tabular}{lcc}
\hline Metodologia & Número de indicações & Percentual \\
\hline EFM - Ecological Footprint Method & 11 & $13,92 \%$ \\
DS - Dashboard of Sustainability - IISD & 10 & $12,66 \%$ \\
BS - Barometer of Sustainability - IUCN & 7 & $8,86 \%$ \\
HDI - Human Development Index - UN & 5 & $6,33 \%$ \\
PSR - Pressure, State, Response - OECD & 5 & $6,33 \%$ \\
DSR - Driven Force, State, Response - CSD & 5 & $6,33 \%$ \\
GRI - Global Reporting Initiative & 4 & $5,06 \%$ \\
GPI - Genuine Progress Indicator & 4 & $5,06 \%$ \\
IWDE - Interagency Working Group on Sustainable Development - US Pres. & 4 & $5,06 \%$ \\
Council on SD & & \\
EIP - European Indices Project - Eurostat & 3 & $3,80 \%$ \\
SOB - System Basic Orientator - Hartmut Bossel - Kassel University & 3 & $3,80 \%$ \\
ESI - Environmental Sustainability Index - World Economic Forum & 3 & $3,80 \%$ \\
CS - Compass of Sustainability & 2 & $2,53 \%$ \\
PPI - Policy Performance Indicator (Holanda) & 2 & $2,53 \%$ \\
DPSIR - Driven, Pressure, State, Impact, Response & 2 & $2,53 \%$ \\
WN - Wealth of Nations - World Bank & 1 & $1,27 \%$ \\
4KM - Four Capitals Model & 1 & $1,27 \%$ \\
MIPS - Material Input Per Service - Wuppertal Institut (Alemanha) & 1 & $1,27 \%$ \\
NRTE - National Round Table on the Environment and Economy - & 1 & $1,27 \$$ \\
Human/Ecosystem Approach (Canadá) & & \\
EnSp - Environmental Space - Wuppertal Institute/Friends of the Earth & 1 & $1,27 \%$ \\
SEEA - System of Integrating Environment and Economic - & 1 & $1,27 \%$ \\
United Nations Statistic Division & & \\
HEI - Human Environment Index & 1 & $1,27 \%$ \\
SM - Swedish Model & 1 & $1,27 \%$ \\
Ecco - Evaluation of Capital Creation Options & 1 & $1,27 \%$ \\
\hline
\end{tabular}

No gráfico 2, podem ser observados os percentuais obtidos pelas principais metodologias. Para efeito de agrupamento, o item FIN (finanças) se refere às metodologias que envolvem principalmente contabilidade ambiental (GPI, WN, ESI). Quanto ao item OUT (outras), este agrupa as metodologias que obtiveram índices de no máximo $5 \%$ no conjunto geral. 
Gráfico 2

Distribuição das indicações entre as ferramentas de avaliação de sustentabilidade

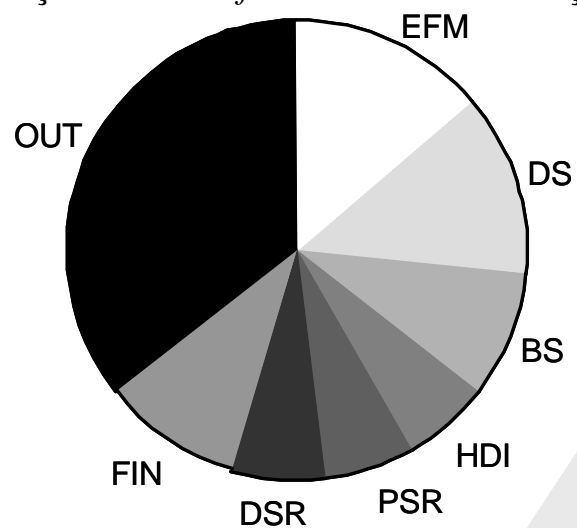

Fonte: o autor

\section{Considerações finais}

Como já observado anteriormente, ocorre uma grande fragmentação de indicações entre as metodologias. Entretanto, as três primeiras ferramentas englobam um percentual semelhante às diversas metodologias agrupadas dentro da classe OUT. Observa-se também que entre as metodologias que ficaram numa posição intermediária, acima de 5\%, a melhor posicionada foi a HDI, que não necessariamente tem relação direta com o conceito de sustentabilidade. Esse fato pode ser explicado pelo grande destaque que recentemente a mídia vem dispensando a esse instrumento. Outro aspecto importante a ser ressaltado é que a metodologia sugerida pela ONU, o DSR, não foi muito lembrada entre os entrevistados, muito embora exista uma iniciativa internacional no sentido de se desenvolver e aplicar essa ferramenta.

Da lista de 18 ferramentas que foi enviada juntamente com o questionário, duas não foram relacionadas pelos entrevistados: EE - Eco Efficiency, do World Business Council on Sustainable Development, e o SPI Sustainable Process Index, do Institute of Chemical Engeneering, da Graz University. Contudo, foram lembradas oito metodologias que não constavam da lista original, que são:

- Global Reporting Initiative - uma iniciativa organizacional, fortemente associada ao conceito de ecoeficiência, desenvolvido pelo WBCSD, e que provavelmente deve substituí-lo;

- Four Capitals Model - iniciativa ligada à área de contabilidade ambiental;

- Compass of Sustainability - relacionada à ferramenta desenvolvida pelo IISD, o Dashboard of Sustainability;

- Environmental Space - desenvolvida pelo Friends of the Earth, juntamente com o Instituto Wuppertal, na Alemanha;

- DPSIR - Driven, Pressure, State, Impact, Response, que é derivado do DSR;

- HEI - Human Environment Index, SW - Swedish Model e o ECCO - Evaluation of Capital Creation Options, que são modelos pouco conhecidos.

As etapas anteriores deste trabalho conduziram às ferramentas de avaliação de sustentabilidade que, na perspectiva dos especialistas em desenvolvimento sustentável, são mais reconhecidas internacionalmente. As três metodologias mais lembradas neste levantamento (Ecological Footprint Method, Dashboard of Sustainability e o Barometer of Sustainability) cobrem mais de um terço das indicações. Nesse sentido, justifica-se a escolha dessas ferramentas bem como sua utilização numa análise comparativa, o que será objeto da próxima etapa deste projeto de pesquisa. 


\section{Referências bibliográficas}

BRÜSEKE, F. J. 0 problema do desenvolvimento sustentável. In: Cavalcanti, C. (Org.). Desenvolvimento e natureza: estudos para uma sociedade sustentável. São Paulo: Cortez, 1995.

CERVO, L. A.; BERVIAN, P. A. Metodologia científica. São Paulo: Makron Books do Brasil, 1996.

CHEVALIER, S., et al. User guide to $\mathbf{4 0}$ community health indicators. Ottawa: Community Health Division, Health and Welfare Canada, 1992.

COSTANZA, R. Ecological economics: the science and management of sustainability. New York: Columbia Press, 1991.

GALLOPIN, G. C. Environmental and sustainability indicators and the concept of situational indicators. A system approach. Environmental Modelling \& Assessment, v.1, p.101-117, 1996.

GOLDSM ITH, E., et al. Blueprint for survival. Boston: Penguin, Harmondsworth \& Houghton Mifflin, 1972.

HAM M OND, A., et al. Environmental indicators: a systematic approach to measuring and reporting on environmental policy performance in the context of sustainable development. Washington, D.C.: World Resources Institut, 1995.

HOBSBAWM, E. A era dos extremos: o breve século XX - 1914-91. São Paulo: Editora Schwarcz, 1996.

HOLING, C. S. (Ed.) Adaptive environmental assessment and management. Chichester: John Wiley \& Sons Ltd., 1978.

IUCN/UNEP/WWF. World conservation strategy: living resource conservation for sustainable development. Gland, Switzerland: International Union for Conservation of Nature and Natural Resources (IUCN); United Nations Environment Programme (Unep); World Wildlife Fund (WWF), 1980.

M CQUEEN, D.; NOAK, H. Health promotion indicators: current status, issues and problems. Health Promotion, v.3, p.117-125, 1988.

MEADOWS, D. et al. The limits to growth. London: Potomac, 1972.

M OLDAN, B.; BILHARZ, S. (Eds.). Sustainability indicators: report of the project on indicators of sustainable development. Chichester: John Wiley \& Sons Ltd., 1997.

MUNASINGHE, M.; M cNEELY, J. Keys concepts and terminology of sustainable development. In: MUNASINGHE, M.; SHEARER, W. (Eds.). Defining and measuring sustainability: the biogeophysical foundations. Washington, D.C.: The United Nations University; The World Bank, 1995.

OECD. Organization for Economic Cooperation and Development: core set of indicators for environmental performance reviews; a synthesis report by the group on the State of the environment. Paris, 1993.

PRONK, J.; UL HAQ, M. Sustainable development: from concept to action. The Hague Report. New York: United Nations Development Programme, 1992.

SACHS, I. Desenvolvimento sustentável, bio-industrialização descentralizada e novas configurações rural- urbanas. Os casos da Índia e do Brasil. In VIEIRA, P. F.; WEBER, J. (Orgs.). Gestão de recursos naturais renováveis e desenvolvimento: novos desafios para a pesquisa ambiental. São Paulo: Cortez, 1997.

TUNSTALL, D. Developing environmental indicators: definitions, framework and issues. Background materials for the World Resources Institute. In: Workshop on Global Environmental Indicators, December 7- 8, 1992, Washington, D.C., World Resources Institute, 1992. (Draft paper).

. Developing and using indicators of sustainable development in Africa: an overview. Prepared for the Network for Environment and Sustainable Development in Africa (NESDA). In: Thematic Workshop on Indicators of Sustainable Development, May 16-18, 1994, Banjul, The Gambia, 1994. (Draft paper).

WORLD COM MISSION ON ENVIRONM ENT AND DEVELOPM ENT. Our common future. Oxford: Oxford University Press, 1987. 